\title{
Quasi-buckling of micromachined beams
}

\author{
Ulf Lindbergt, Jan Söderkvistt, Theo Lammerinkł and \\ Miko Elwenspoekł \\ † Department of Technology, Uppsala University, Box 534, S-751 21 Uppsala, \\ Sweden \\ $\ddagger$ Department of Electrical Engineering, University of Twente, PO Box 217, 7500 \\ AE Enschede, Netherlands
}

\begin{abstract}
Buckling of structures with imperfections, quasi-buckling (QB), is studied. At the bifurcation load, these structures show a smooth transition into one of the stable postbuckling equilibrium states, instead of the traditional sudden change in deflection characteristics. Of course, QB structures can show the classical snap-through buckling behaviour, i.e., a sudden change of postbuckling equilibrium state. Here, the $\mathrm{QB}$ is described with a generalized temperature, $T_{\mathrm{g}}$, representing the compression of the structure. Imperfections and distributed deflection loads are represented by a generalized pressure, $p_{\mathrm{g}}$. Experiments on micromachined beams, exposed to heating $\left(T_{g}\right)$ and to a Lorentz force $\left(p_{g}\right)$, verify that the $Q B$ phenomena can efficiently transfer a longitudinal stress into a transversal deflection, with a scale-factor depending on both $T_{\mathrm{g}}$ and $\mathrm{Pg}_{\mathrm{g}}$. Also, a $\rho_{\mathrm{g}}$ "guided snap-through can be eased by reducing $T_{g}$ during the snap-through.
\end{abstract}

\section{Introduction}

Beams that become unstable under compressive loads were studied as early as the 18th century by Euler. The Euler equations for the buckling load of beams are well established. According to the ideal theory, the structure can choose any of several stable and unstable states above the critical buckling load. Thus, the postbuckling behaviour, including the direction of the deflection is unpredictable above this load. In reality, small geometric imperfections and unsymmetric loads determine the postbuckling behaviour. Although buckling is often undesirable [1], it can be used as a feature by purposely introducing imperfections, e.g., in actuators, switches [2,3] and diagnostic tools [4]. Previous work on micromechanical buckling switches mainly uses the traditional abrupt change of equilibrium postbuckling state (snap-through) of prestressed beams exposed to a transverse guiding electrostatic force. The buckling phenomena can be expected to be more noticeable for micromachined structures since the fabrication often introduces large internal compressive stress.

\section{Quasi-buckling}

Classical buckling is initiated by a compressive average stress. The corresponding average strain $\epsilon_{\mathrm{a}}$, can be described with an easily interpretable generalized temperature, $T_{\mathrm{g}},\left(T_{\mathrm{g}}=\epsilon_{\mathrm{a}} / \alpha, \alpha\right.$ is the thermal expansion coefficient). Introduce a normalized temperature, $x$, related to the classical Euler buckling load, $T_{\mathrm{g}}$. This gives for the support condition of the beam in figure 1 :

$$
\begin{gathered}
x=\frac{T_{\mathrm{g}}}{T_{\mathrm{c}}}=\frac{\epsilon_{\mathrm{a}}}{\alpha T_{\mathrm{c}}} \\
T_{\mathrm{c}}=\frac{\pi^{2} t^{2}}{3 \alpha L^{2}}
\end{gathered}
$$

where $T_{\mathrm{g}}$ and $T_{\mathrm{c}}$ are measured relative to the temperature at which the surface is stress free. The average temperature of the beam in figure 1 is controlled via a thin film resistor, and equals

$$
T_{\mathrm{g}}=\frac{U^{2} L}{8 b t \lambda R}
$$

if energy dissipates only via the mounting ( $U, \lambda$ and $R$ are the applied voltage, the heat conductivity and the resistance of the heater) [5].

The addition of a guiding transversal force makes it possible to describe imperfections, dissymmetries, and external transversal loads. It can often be represented by a generalized pressure, $p_{\mathrm{g}}$, which is also, in a first approximation, appropriate for small changes in point loads. Let $\delta_{0}$ denote the $p_{\mathrm{g}}$-generated transversal defiection at the centre of the beam in figure 1 when no compressive stress is present, i.e., when $x$ equals zero. The increase in transversal amplitude when a compressive load is added can analytically be 


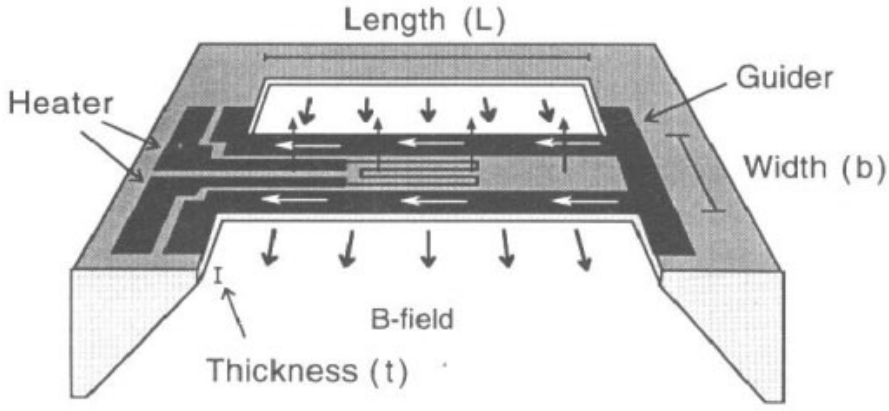

Figure 1. Silicon structure used for testing of controlled $Q B$. The electric current $I$ (white arrows) and the magnetic field $B$ (thick dark arrows) determine the Lorentz force $F_{L}$ (thin dark arrows). The small loop current used for the heater does not result in a Lorentz force.

approximated with the following two expressions $[2,6]$, except near buckling $(x \approx 1)$ :

$$
\begin{aligned}
& \delta(x<1)=\frac{\delta_{0}}{1-x} \\
& \delta(x>1)=\frac{2 t}{\sqrt{3}} \sqrt{x-1} \equiv k \delta_{0} \sqrt{x-1}
\end{aligned}
$$

and

$$
\delta_{0}=\frac{p_{\mathrm{g}} L^{4}}{32 E t^{3}} \quad k=\frac{64 E t^{4}}{p_{\mathrm{g}} L^{4} \sqrt{3}}
$$
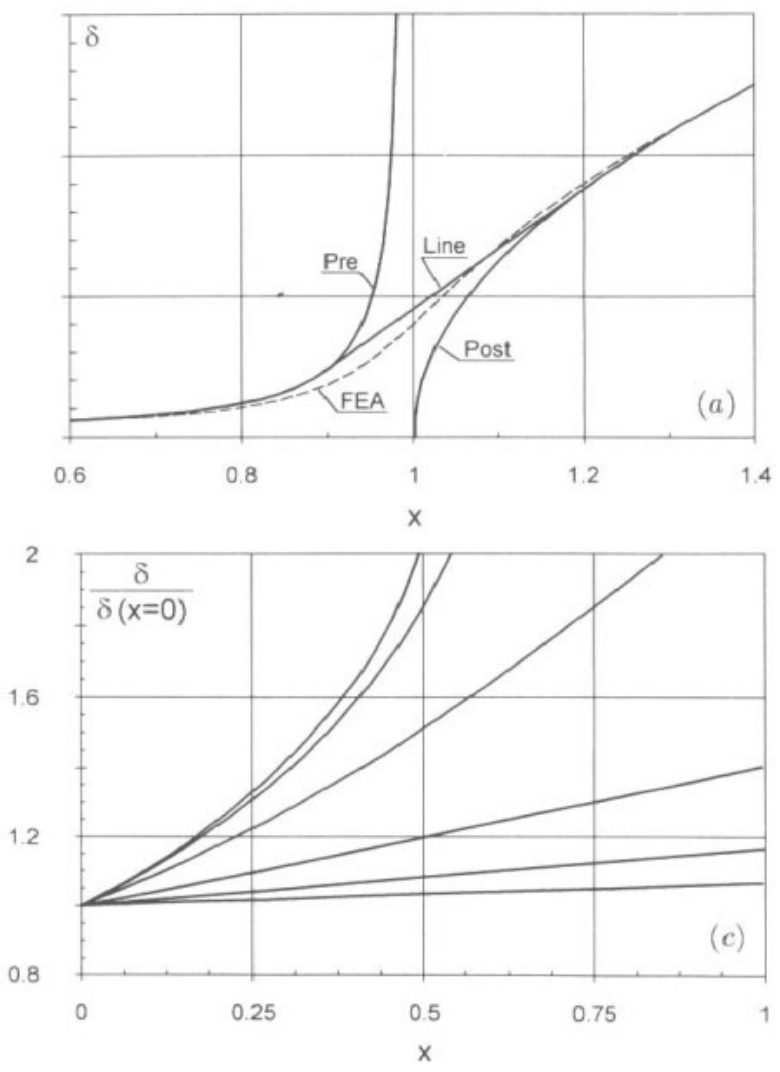

Equation (3) divides the problem into prebuckling and postbuckling, as illustrated in figure $2(a)$. The intermediate region can be approximated with the straight tangential line

$$
\delta=\frac{(x-1) \delta_{0} k^{4 / 3}}{4}+\delta_{0} k^{2 / 3}
$$

with slope $\delta_{0} k^{4 / 3} / 4$, between the points

$$
\begin{aligned}
& \left(\begin{array}{l}
x \\
\delta
\end{array}\right)=\left(\begin{array}{c}
\max \left(0,1-2 k^{-2 / 3}\right) \\
\delta_{0} k^{2 / 3} / 2
\end{array}\right) \\
& \left(\begin{array}{l}
x \\
\delta
\end{array}\right)=\left(\begin{array}{c}
1+4 k^{-2 / 3} \\
2 \delta_{0} k^{2 / 3}
\end{array}\right) .
\end{aligned}
$$

This results in the Jeflections $\delta=\delta_{0} k^{2 / 3}$ at the classical buckling load, $T_{c}$. The dashed curve in figure $2(a)$ is obtained via a non-linear finite element analysis (FEA), and can be considered as the exact solution. Note that $\delta$ depends mainly on the transversal Ioad for $x \ll 1$ (prebuckling) but only on the compressive load when $x \gg 1$ (postbuckling). This makes it possible to transfer a small longitudinal motion into a large transversal in a controlled way. Figures $2(b)$ and $2(c)$ show FEA-calculated deflection curves as a function of compressive load, for various values of the guiding force. Clearly, the traditional sudden change in
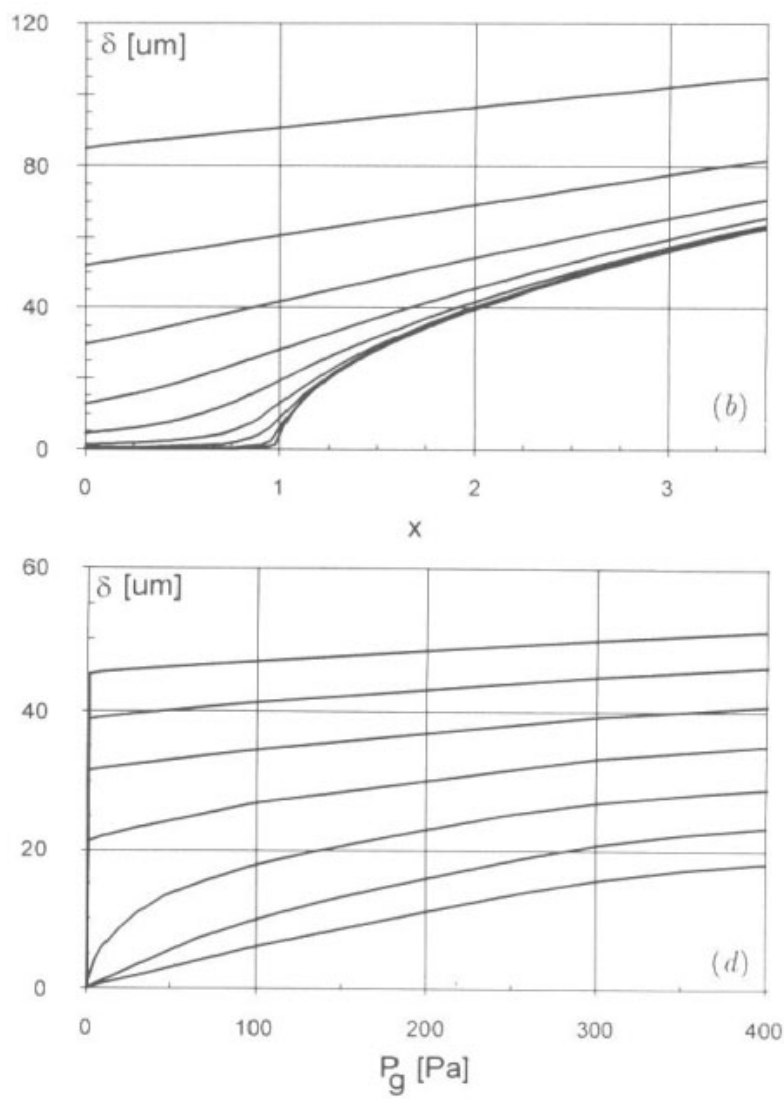

Flgure 2. Mid-point deflections for the silicon beam in figure 1, with $L=10 \mathrm{~mm}, b=1.2 \mathrm{~mm}, t=34 \mu \mathrm{m}$ and $E=167 \mathrm{GPa}((110))$. The solid curves in figure 2(a) are based on equation (3a) with $\mathrm{p}_{\mathrm{g}}=10 \mathrm{~Pa}$, while the other have been determined with FEA (ANSYS). The curves are, from top to bottom, for $P_{g}=$ $10 \mathrm{k}, 3 \mathrm{k}, 1 \mathrm{k}, 300,100,30,3$ and $1 \mathrm{~Pa}$ (figure $2(\mathrm{~b})$ ), $\rho_{\mathrm{g}}=30,100,300,1 \mathrm{k}, 3 \mathrm{k}$ and $10 \mathrm{k}$ Pa (figure 2(c)) and $T_{9}=34,29,24,19,14,9$ and $4{ }^{\circ} \mathrm{C}$ (tigure $2(d), T_{c}=14.7^{\circ} \mathrm{C}$ ). 
deflection characteristics at $T_{\mathfrak{c}}$ is less pronounced if $p_{\mathfrak{g}}$ is larger (quasi-buckling), e.g., due to imperfections. A sometimes unexpected consequence is that the amplification factor in figure 2(c) is largest when the imperfections are small, which may result in unexpected and unpredictable behaviour well below $T_{\mathrm{c}}$ if the kind and magnitude of imperfections are known. The FEA curves in figure $2(d)$ show the increase in $\delta$ with the transversal load, for various levels of the compressive prestress.

The dynamic spring constants can be determined from equation (3) or from the slope of the curves in figure 2. The smallest transverse spring constant, $\Delta p_{\mathrm{g}} / \Delta \delta$, is found at low values of $T_{\mathrm{g}}$ and $p_{\mathrm{g}}$, and smallest compressive spring constant, $\Delta T_{\mathrm{g}} / \Delta \delta$, at $T_{c}$. The linear analytic expressions in equation (3) give a reliable first estimate of the transversal behaviour. However, a saturation occurs if the deflection approaches half the thickness of the beam, which is clearly visible in figure $2(b)$ for $\delta(x=0)$. The deflection there is not proportional to $p_{\mathrm{g}}$ for large $p_{g}$. FEA should be considered in this case. Note that other buckling situations can be studied by using the appropriate expressions for $\delta_{0}$ and $T_{\mathrm{c}}$, e.g., found in [7].

\section{Experiment}

Experiments on the structure in figure 1 have been carried out to show that controlled $\mathrm{QB}$ is achievable with standard micromachining techniques. The structures were fabricated from (100) wafers using a $\mathrm{Si}_{3} \mathrm{~N}_{4}$ protecting layer, $\mathrm{KOH}$ etch and evaporated $\mathrm{Cr} / \mathrm{Au}$ electrodes. The deflection of the beam was observed using a reflected light beam. A current-controlled Lorentz force, $F_{\mathrm{L}}$, generated the guiding force $\left(p_{\mathrm{g}}=F_{\mathrm{L}} / b L=B I / b\right)$ in our experiments. An electrostatic guiding force would have resulted in several disadvantages when exploring $\mathrm{QB}$ :

(i) mono-directionality

(ii) deflection dependence

(iii) risk of electrostatic collapse.

Size can be reduced by using one magnetic field to generate Lorentz forces for several beams. The square voltage dependence of the heat-generated compressive force implies that a linear dependence of the deflection on the heating voltage can be expected for large postbuckling deflections, if saturation is neglected (cf. equation (3a)). The temperature was increased slowly to get a well-defined and easily interpreted steady state. Faster changes generate an additional AC thermal field localized to the electrode area, with a thermal time-constant $(\approx 100 \mathrm{kHz})$ mainly determined by the thickness of the beam.

A compressive $14{ }^{\circ} \mathrm{C}$ buckling load was measured by increasing the heating voltage until the deflection increased rapidly. No guiding Lorentz force was used. The rapid increase in deflection indicates that the imperfections in the structure were small. The importance of the imperfections was measured by repeating the experiment with a non-zero constant guiding Lorentz force. The minimum guiding force needed to change the direction of the $Q B$ from upward to downward corresponded to $40 \mathrm{~Pa}$. This corresponds to an imperfection-generated upwards deflection of $\delta_{0}$ $=1.9 \mu \mathrm{m}$.

The inaccuracies in the measurements are estimated to be $20 \%$. Possible imperfections in the structure are due to:

(i) a slight yield in the mounting which can give the beam a tendency to bend upwards

(ii) stress in the protecting and electrode layers

(iii) etching-generated geometric imperfections.

An inaccuracy in the model is the treatment of the mounting as an ideal heat sink.

\section{Discussion}

The magnitude and direction of quasi-buckling of beams can be controlled continuously by simultaneously using compressive and transversal loads. For instance, a compressive force can reduce the magnitude of the transversal force needed for changing stablepostbuckling-state snap-through. This is useful in (arrays of) non-volatile memory cells and digital light reflectors. Also, the dependence of the transversal deflection on compressive loads smooths the behaviour of the buckling beams, and makes it possible to transfer a small longitudinal motion into a large transverse. It is even possible to vibrate the beam in a bidirectional and continuous way by using a time-varying compressive force aided by a small transverse guiding force.

\section{Acknowledgments}

This work was made possible by grants from the Swedish National Board for Industrial and Technical Development (NUTEK).

\section{References}

[1] Ding X and Ko W H 1991 Buckling behaviour of boron-doped $\mathrm{p}^{+}$silicon diaphragm Proc. Transducers 91 (San Francisco, CA, 1991) (New York: IEEE) pp 201-4

[2] Hălg B 1990 On a micro-electro-mechanical nonvolatile memory cell IEEE Trans. Electron Devices 37 2230-6

[3] Huff M A, Nikolich A D and Schmidt M A 1991 A threshold pressure switch utilizing plastic deformation of silicon Proc. Transducers 91 (San Francisco, CA, 1991) (New York: IEEE) pp 177-80

[4] Guckel H, Burns D, Rutigliano C, Lovell E and Choi B 1992 Diagnostic microstructures for the measurement of intrinsic strain in thin films $J$. Micromech. Microeng. 2 86-95 
$\mathrm{U}$ Lindberg et al

[5] Lammerink T, Elwenspoek M, Ouwerkerk Rv,

Bouwstra S and Fluitman J 1990 Performance of thermally excited resonators Sens. Actuators A21-23 $352-6$
[6] Timoshenko S and Gere J 1961 Theory of Elastic Stability (New York: McGraw-Hill)

[7] Young W C 1989 Roark's Formulas for Stress \& Strain 6th edn (New York: McGraw-Hili) 